\title{
Healthy food in school canteens, policy directions and deviations: A cross sectional study from Sri Lanka
}

\author{
Manuj C. Weerasinghe ${ }^{1, *}$, Samanthi Bandara ${ }^{2}$, Munsif Sanoon ${ }^{3}$ \\ ${ }^{1}$ Senior Lecturer, Dept. of Community Medicine, University of Colombo, Sri Lanka, ${ }^{2}$ Research Officer, Institute of Policy \\ Studies of Sri Lanka, 3Medical Officer, Ministry of Health, Sri Lanka
}

*Corresponding Author:

Email: Manuj@commed.cmb.ac.lk

\begin{abstract}
Introduction: Sri Lanka introduced school canteen guidelines in 2007 to promote healthy food choices and eating habits among school children. This initiative intended to combat malnutrition among school children through provision of nutritious, culturally acceptable food at affordable prices within the school premises. A formal assessment has not been done on the implementation of those guidelines for last seven years. The objective of this study was to assess the availability of healthy food items in school canteens and explore perceptions of providers on adherence to the guidelines.

Materials and Methods: A cross sectional study was conducted in government schools in two districts. A mix methodology was employed. An observational check list was used to quantitatively assess the availability of healthy food items according to stipulated guidelines. This was followed by a qualitative inquiry to explore perceptions of school principals and canteen operators on adherence to the guidelines using in-depth interviews.

Results: Only two out of ten food items encouraged in the guidelines were available in $50 \%$ of the school canteens. In contrast, five out of nine items prohibited or discouraged were available in half of the canteens. Also $25 \%$ of canteens sold carbonated drinks that are totally prohibited. Differential understanding of healthy versus unhealthy, current food habits of students, present method of selecting canteen operators and other external factors contributed negatively.

Conclusion: The rigor of implementation was sub optimal at present due to many reasons. Those obstacles can be addressed through coordinated efforts to transform canteen towards a nutrition promoting setting.
\end{abstract}

Keywords: School health, Child and adolescent nutrition, Health promotion, Health policy, Food habits.

\section{Introduction}

When school food environment can be made healthy, it influences students to make healthy food choices and develop healthy eating habits. ${ }^{1}$ Many initiatives have been taken on this direction during the last decade and a half in several countries. Healthy food and drink policy of Western Australian Department of Education and Training, a legislative enactment in a state in Brazil to lay down criteria for provision of snacks/ drink services in educational institutions and healthy school canteen program in Netherlands are few examples. ${ }^{1,2,3}$ The World Health Organization resolution WHA 57.17 in 2004 provided an impetus for initiatives on healthy diet throughout the world. ${ }^{4}$ The most comprehensive approach on healthy school canteen could be seen in the program initiated by the Australian government. The directives from the National Healthy School Canteens Project provided clear guidelines for food selection, food safety, food handling, training, information system and monitoring and evaluation of the programme. $^{5}$ This provided sufficient guidance and technical support to the school authorities.

Initiation of a dialogue on a healthy school canteen policy in Sri Lanka during 2006/2007 period and subsequent directives on maintaining a school canteen that promote healthy nutrition among school children could be considered as part of the global push towards healthy diet. The discussions aimed to combat malnutrition among school children through provision of nutritious, culturally acceptable food at affordable prices within the school premises. In addition enhancing food hygiene, promotion of healthy dietary habits, establishment of child friendly service and improvement in facilities of school canteens were expected through the guidelines. Based on the draft policy document a circular was issued in 2007 (MOE circular 2007/2) to formalize the functioning of school canteens. A follow up circular was issued in 2011 (MOE circular 2011/3) to strengthen the initiative. Further, a direction was issued to schools (MOE circular 2007/21) for establishment of health promotion committees that could engage in enhancing and monitoring of healthy activities in schools including nutrition of students. The guidelines issued in 2007 focused on seven points for maintaining a school canteen. Three more points were added in 2011. Among those guidelines, hygienic preparation of food, appropriate nutritional value, improving opportunities to buy healthy foods in school canteen and prohibiting unhealthy food were prominent.

\section{School Canteens in Sri Lanka}

Sri Lanka has over 9000 schools across the country. For administrative purposes the country is divided into districts and a district is divided into several educational zones. Each educational zone has many educational divisions managed by a divisional director. Within an educational division schools are categorized into 4 types. According to the categorization; Type $1 \mathrm{AB}$ schools have 
classes up to Advanced Level including science, arts and commerce subjects, Type 1C schools have classes up to Advanced Level but only arts and commerce subjects, Type 2 schools have classes only up to grade 11 and Type 3 schools have classes only up to grade 08 . Type 3 schools are small with few students and less facilities. Advanced Level classes in school curricular in Sri Lanka are similar to the high schools in other countries. The school canteen is outsourced to a private party. This is done according to government financial procedures. Biding documents contain minimum requirements for a canteen operator and a list of food items that need to be provided. This list of food items prescribed by the education ministry contains the minimum that should be available in the canteen. The operator has the freedom to include additional food items that they wish to provide with approval. Biding documents specify a minimum rate a bidder has to pay to the school authorities based on 'student per head'. The highest bidder fulfilling the minimum requirements is awarded the tender in an annual basis. Selection of canteen operators is done by the zonal education office without any consultation with the schools.

Eight years has passed since the preparation of guidelines to strengthen school canteens. The objective of this study was to determine the availability of healthy food items in school canteens as prescribed by the guidelines and to explore the perception of providers on adherence to the guidelines in two districts of Sri Lanka.

\section{Materials and Methods}

A cross sectional study was conducted in government schools in two districts in the Western province of Sri Lanka. A mix methodology was used; to quantitatively assess the availability of healthy food items according to guidelines and to explore perceptions on adherence through in-depth interviews. We selected schools according to the above mentioned classification excluding type 3 schools with fewer students. There are four educational zones in each of the districts, Colombo and Gampaha. A stratified sampling method was adopted to secure adequate representation of $1 \mathrm{AB}, 1 \mathrm{C}$ and type 2 schools. In the first step two educational zones of each district were selected by simple random sampling. Then, two educational divisions from each of the selected educational zones were chosen using same method. Hence, eight educational divisions from both districts were selected. The type of school was considered as the 'stratum' and three schools were selected from each stratum in an education division, making the final sample of 72 schools. Following the quantitative assessment, twelve in-depth interviews, six each with principals of schools and canteen operator's, were conducted to inquire their perceptions on the adherence to guidelines. Twelve schools for in-depth interviews were selected based on the type of school, medium of instruction and the status of the canteen during the preliminary observation.
A check list was used to record the observations in the school canteens for availability of different food items. The check list consisted of food items prescribed in the guidelines as healthy and those prohibited for sale or restricted as unhealthy. At present Sri Lanka has not developed a widely accepted food classification based on nutrition profiling. Hence, this assessment was solely based on the directions provided in the guidelines and the food list provided to the canteen operators by the ministry. A flexible format guided the in-depth interviews.

Four teams of field investigators were trained for data collection. Data collection was carried out during the month of September 2014. Schools were only informed that a study would be conducted during the latter half of the year. No prior notification was given on the day or the exact nature of data collection to prevent possible bias due to prior preparation. All efforts were taken to complete data collection in adjacent schools within the same day. Data collection in an education zone was completed within a week to minimize contamination effects. The data collectors visited a school close to the mid-day break when the canteen is fully functional. While the data collectors were engaged in the canteen carrying out their observations, the principal investigator with the support of coinvestigators conducted the in-depth interviews. Same weightage was given to all the types of schools in the analysis, as all school canteens is expected to have similar standards. Availability of food items considered to be healthy and unhealthy according to the guidelines is presented as counts and percentages.

Qualitative data was analyzed inductively through an iterative process of reading, coding, recoding, displaying, reducing, and summarizing into themes manually. Comparison across interviews and identification of emerging themes provided the opportunity to unearth core issues related to adherence and deviations from the guidelines. The research protocol, study instruments and the procedure was approved by the Ethics review committee of the Faculty of Medicine, University of Colombo. School authorities granted permission to observe the school canteens and record data. Informed verbal consent was obtained from the school principals and the canteen operators prior to the in-depth interviews.

\section{Results}

Results are presented in two parts. School characteristics and availability of food items are presented in tabular form followed by qualitative analysis of in-depth interviews. Table 1 presents distribution of schools according to the districts, zones and the type of school. In total 71 schools were studied from the initial sample of 72 . Of the 71 schools only 68 schools had a canteen. 
Table 1: Selection of Schools

\begin{tabular}{|l|c|l|l|l|}
\hline District & $\begin{array}{c}\text { Number of } \\
\text { Educational } \\
\text { Zone }\end{array}$ & $\begin{array}{c}\text { Number of } \\
\text { Educational } \\
\text { Division }\end{array}$ & $\begin{array}{c}\text { Type of } \\
\text { School }\end{array}$ & $\begin{array}{c}\text { Number } \\
\text { of Schools }\end{array}$ \\
\hline Colombo & 2 & 4 & $1 \mathrm{AB}$ & 12 \\
\hline & & & $1 \mathrm{C}$ & 12 \\
\hline & & & Type 2 & 12 \\
\hline Gampaha & 2 & 4 & $1 \mathrm{AB}$ & 12 \\
\hline & & & $1 \mathrm{C}$ & 12 \\
\hline & & & Type 2 & 12 \\
\hline Total & \multicolumn{3}{|l}{} \\
\hline
\end{tabular}

Availability of foods and beverages encouraged in the canteen guidelines and those prohibited or discouraged are given in the Tables 2 and 3 respectively. It was found that only two out of ten food items encouraged in the guidelines were available in $50 \%$ of the school canteens. In contrast, five out of nine items prohibited or discouraged were available for sale in over $50 \%$ of canteens. Twenty five percent of canteens also sold carbonated drinks that are totally prohibited.

Table 2: Food and Beverages Encouraged to be Sold in the School Canteen according to the Guidelines $(\mathbf{n}=68)$

\begin{tabular}{|l|c|c|}
\hline \multirow{2}{*}{ Food Item } & \multicolumn{2}{|c|}{ Available } \\
\cline { 2 - 3 } & Frequency & Percent (\%) \\
\hline Rice & 53 & 77.9 \\
\hline Chick peas & 31 & 45.6 \\
\hline Cowpea & 10 & 14.7 \\
\hline Rice flour string hopper & 43 & 63.2 \\
\hline Rice flour hoppers & 23 & 33.8 \\
\hline Dosai (Ulundu flour) & 32 & 47.1 \\
\hline Fresh juice & 9 & 13.2 \\
\hline Fresh milk & 6 & 8.8 \\
\hline Pasteurized milk & 21 & 30.9 \\
\hline Kola kanda (Leafy porridge) & 5 & 7.4 \\
\hline
\end{tabular}

Table 3: Food and Beverages Discouraged or Prohibited to be sold in the School Canteen according to the Guidelines $(\mathbf{n}=68)$

\begin{tabular}{|l|c|c|}
\hline \multirow{2}{*}{ Food Item } & \multicolumn{2}{|c|}{ Available } \\
\cline { 2 - 3 } & Frequency & Percent (\%) \\
\hline Wheat flour bread & 35 & 51.5 \\
\hline Wheat flour Roti & 36 & 52.9 \\
\hline Pastry & 39 & 57.4 \\
\hline Deep fried short eats & 55 & 80.9 \\
\hline Chip items & 33 & 48.5 \\
\hline Chocolates & 14 & 20.6 \\
\hline Toffees & 39 & 57.4 \\
\hline Carbonated drinks & 17 & 25.0 \\
\hline Malted drinks & 10 & 14.7 \\
\hline
\end{tabular}

\section{Analysis of qualitative data}

The qualitative data is presented under four broad themes; perception on healthy versus unhealthy food, awareness on guidelines, food habits, preferences and profit, and barriers for adhering to guidelines. Emerging subthemes converged on those four broader themes with linkages across the themes.

\section{Perception on healthy versus unhealthy food}

The school canteen guidelines intended to inculcate healthy food habits among children through an enabling school environment. The understanding of healthy versus unhealthy food in the minds of the providers is crucial to achieve this goal. Although both principals and the canteen operators recognized the importance of proper nutrition as a key factor for successful attainment 
in education, there were differences in the understanding on the concept of healthy food. Most school principals were comfortable categorizing traditional foods as healthy in a blanket manner without considering the nutritional content and value of those. The notion that traditional food is better in every aspect was held as an unchallengeable fact. They tend to believe drifting from traditional diet to modernized food intake as the sole cause of malnutrition and disease, without critically looking at the actual content in a diet. The canteen operator's understanding was almost limited to believing rice flour based products as healthy and wheat flour based products and sweets as unhealthy. Method of food preparation and other ingredients were not considered. As the guidelines did not elaborate on the concept of healthy food beyond a list of examples, the directions themselves provided limited insights on this issue.

\section{Awareness on guidelines}

Majority of the principles have seen the canteen guidelines. Although principals had general understanding on the content of the circular, most of them were not aware on the specific issues addressed on selection of food items such as high sugar, salt and fat containing food and beverages. All of them knew that school canteen should not have soft drinks and refrain from selling sweets, but were less aware on zero calorie and junk food. They also knew that wheat based food items needed to be restricted and rice and rice flour based food should be encouraged. Canteen operators could mainly recollect the instructions imposed on restricted food items but little on the promotive aspects of the guideline.

\section{Food habits, preferences and profit}

Both the principals and canteen operators observe that majority of the students prefer food items categorized as oily, sugary or unhealthy in broad terms. Main demand of student is for pastries, fried items, and sweets. The least demand is for food made up of pulses which are recommended in the guidelines as healthy options. Principals and canteen operators both insisted that, it is extremely difficult to convince students to have healthy options available in the canteen. Serving rice and curry is promoted in the guidelines as a suitable food item. However, according to the canteen operators and our observations the demand is for 'fried rice' and not for traditional rice and curry menu which is considered to be healthy. Hence, rice as a food item is sold more often as fried rice with minute amount of fried vegetables. Apart from the food items listed in the tender documents, many other items were sold in canteens. Canteen operators revealed that additional food items have a better demand than those listed by the ministry. Profit margin for those additional food items is higher than the mandatory ones. Further, purchasing capacity of students is limited; hence, they compel to buy food items that are affordable to them. Responding to the situation, canteen operators take an effort to provide food items within the reach of students. It was found that food items claimed as unhealthy are less expensive, thus more affordable to the students. Food items considered as healthy such as pulses, leafy porridge and red rice are generally consumed by teachers.

\section{Barriers for adhering to guidelines}

School principals view food habits of the students to be the main barrier to implement healthy canteen guidelines. They attribute home based food culture, upbringing of children at home, affordability and attitudes of parents on the present food habits of students. The principals pointed out that school cannot change the existing food habits of students in isolation. Students stay only six hours in school and have all three main meals at home. They emphasizes parents should take the lead in changing it. School can only facilitate the change.

Both principals and the canteen operators pointed that shops, bakeries and cafes close to the school sell food items banned or restricted within the school. Therefore, students have easy access to those food items on their way to school and also after school. Many parents buy food from those shops in the morning for the students when they request. Canteen operator's make this situation a point to justify their own actions of selling unhealthy food within the school. School authorities do not have any jurisdiction over the activities happening beyond the school premises. Hence, the implementation of the guidelines in the school canteens is effectively negated.

Awarding of the tenders for operating school canteens is totally handled by the zonal education office. Principals claimed that competitors offer higher bids to win the tender without considering the actual business opportunities in the school canteen. The fluctuation in the student numbers during the year, their absenteeism or their actual use of the canteen is not considered when selecting the bids. The canteen operator should pay the stipulated amount to the school in a daily basis. The principals admit only a proportion of students use the canteen in a regular basis. The selected canteen operator only realizes the ground situation when they find it difficult to achieve the sales to accommodate the costs when adhering to guidelines. The canteen operator at this stage either abandons the agreement and leave or they would sort to other means of increasing their income. If the school wants to continue the canteen, principle has to overlook some of the deviations from the agreement. Hence, all principals expressed that it is not possible to adhere to stipulated guidelines fully unless the current procedure of selecting a canteen operator is changed. Hence, the canteen operators have flexibility to manipulate the situation to evade restrictions and maximize profits. 


\section{Discussion}

Children as well as parents generally believe that anything permitted in the school is inherently healthy. ${ }^{6}$ Further, evidences suggest that provision of unhealthy foods in canteen led to the belief that such products are appropriate for regular consumption. Hence, it is seen food environment in school as a useful strategy to promote healthy diet. $^{7}$ One of the objectives of the guidelines is to provide healthy food in school canteens and to promote desirable food habits among students to improve nutritional status. The findings of this study revealed a selective implementation of the guidelines in relation to the selection and availability of food items. We found several reasons for non-adherence of school canteen guidelines.

The ambiguity on the understanding of the terms, healthy and unhealthy food within the minds of the providers, is a key issue for non-adherence. The guidelines themselves had not adequately addressed this issue. Despite the work done globally to define and simplify the healthy versus unhealthy food in practical terms, ${ }^{8,9}$ the present guidelines issued in Sri Lanka has failed to elaborate on this important element. Simply providing a list of examples without a systematic approach to categorize food items, left individual school administrator or canteen operator freedom for own interpretations based on their knowledge and preferences. In contrast the Western Australian model, that is proved to be functioning satisfactorily, relies on "traffic light categorization" of food items that provide objective direction for both the providers and the consumers. ${ }^{2}$ This allows implementing a homogenous system across the schools and a basis for regular monitoring.

Having higher number of unhealthy food options on school canteen menu was seen as a barrier for healthy eating. ${ }^{6}$ When several alternatives are available, there is a tendency for children to resort to sub optimal choices in relation to nutritive value. It is well established that children will prefer fatty-sugary snack foods to more nutritious food if available. ${ }^{10}$ Also the demand induces the supply of unhealthy food unless suitable alternatives are provided. Those observations have led recommendations to reduce the quantity of competitive foods that act as a deterrent to healthy eating in school canteens. ${ }^{6,11}$ In this study, it was also found that healthy alternatives offered to students in the canteen have failed to create enthusiasm on healthy eating. Hence, the healthy options provided at present were not attractive enough to guide students to change their preferences. In addition, parents preferred convene foods that can be easily carried to school. ${ }^{10}$ In school it is likely that students also prefer those food items partly due to the convenience of consuming during a short break apart from their preference for taste.

Despite the ban on carbonated drinks, it was found that one fourth of canteens still sell them. A previous study done in Colombo schools also reported that all except one $(n=65)$ in their sample sold soft drinks in the canteen. ${ }^{12}$ They further reported $82 \%$ of adolescents consumed sugar sweetened soft drinks one or more times in a week. This provides evidence for the existing demand for carbonated beverage among school going children. In comparison, leafy porridge and fresh juice were sold in only a small number of canteens and with poor demand from students.

The driving forces behind school canteen sales are found non-nutritive. ${ }^{10}$ School canteens exist for profits and fast foods are considered to be the most profitable items. Thus, to sustain the canteen economically viable, canteen operators have drifted from the guidelines to some extent. Outsourcing the canteen to private operators and emphasizing canteen as profit making ventures is identified as a major obstacle for healthy food promotion. Hence, changes in the current logistic arrangements of school canteen are necessary, recognizing this as a future investment on child health than a profit making ventures. ${ }^{11}$ This suggests the present Sri Lankan system of outsourcing school canteen need to be reoriented from a business model towards a welfare model.

One of the key obstacles expressed by principals and canteen operators for maintaining a healthy canteen was the presence of food stalls close to the school that sell unhealthy food. It was found that majority of the schools had food establishments in the vicinity including outlets of corporate food chains. Majority of those offer fast food and carbonated drinks which are restricted in the school premises. Hence, school children have access to unhealthy food items and exposed to unhealthy advertising on their way to school. This reminds the need for wider involvement beyond the school premises to instil healthy behaviours among school children.

\section{Conclusions}

School Canteen policy intended to improve healthy eating habits and nutrition of school children. However, having a canteen policy per se does not guarantee that intended outcomes will be achieved. The rigor of implementation needs to be at an optimal level. Framing the guidelines without detailed instructions for successful implementation, limited attention paid for building an environment conducive for implementation within the school and factors beyond the control of school contributed for the deviations. Prohibiting caloric foods and restricting their advertising per say is unlikely to change the food habits of children. It is paramount to improve the understanding of the school authorities and the canteen operators on healthy food for proper implementation. Further, obtaining cooperation of parents, students and, transforming the concept of school canteen from a business entity to a long term investment for healthy living is necessary.

Abbreviations: MOE-Ministry of Education 


\section{Competing interest}

Authors do not have any competing interest on the study or the publication

\section{Author contribution}

MW conceptualized the study, developed study design, field work, data analysis and writing the draft. SB contributes to study design, filed work and writing the draft. MS contributed to study design, field work and data analysis.

\section{Acknowledgements}

We would like to acknowledge UNICEF for funding the study and Institute of Policy Studies, Sri Lanka for providing logistical and other support to carry out the study.

\section{References}

1. Mensink F, Schwinghammer SA, Smeets A. The healthy school canteen programme: a promising intervention to make the school food environment healthier. J Environ Public Health. 2012;2012:1-8. doi:10.1155/2012/415746.

2. Pettigrew S, Pescud M, Donovan RJ. Outcomes of the West Australian school healthy food and drink policy. Nutrition \& Dietetics. 2012;69(1)20-25. doi:10.1111/j.17470080.2011.01564.x.

3. Gabriel CG, Vasconcelos Fde A, Andrade DF, Schmitz Bde A. First law regulating school canteens in Brazil: evaluation after seven years of implementation. Arch Latinoam Nutr. 2009;59(2):128-38.

4. World Health Organization. Global Strategy on Diet, Physical Activity and Health. Fifty Seventh World Health Assembly WHA57.17. Geneva, Switzerland: 2004. http://www.who.int/dietphysicalactivity/strategy/eb11344 /strategy_english_web.pdf.Accessed 31 Dec 2014.

5. Department of Health, Australian Government. 2010 National Healthy School Canteens Guidelines. Department of Health. http://www.health.gov.au/internet/main/publishing.nsf/Co ntent/phd-nutrition-canteens. Accessed 31 Dec2014.

6. Hesketh K, Waters E, Green J, Salmon L, Williams J. Healthy eating, activity and obesity prevention: a qualitative study of parents and child perception in Australia. Health Promot. Int. 2005;20(1):19-26. doi:10.1093/heapro/dah503.

7. Bell AC, Swinburn BA. School canteens: using ripples to create a wave of healthy eating.Med J Aust. 2005;183(1):5-6.

8. Lobstein T, Davies S. Defining and labeling 'healthy and 'unhealthy' food. Public Health Nutr. 2009;12(3):331-40. doi:10.1017/s1368980008002541.

9. Drescher LS, Thiele S, Mensink GB. A new index to measure healthy food diversity better reflects a healthy diet than traditional measures. J Nutr. 2007;137(3):64751.

10. Bell AC, Swinburn BA. What are the key food groups to target for preventing obesity and improving nutrition in schools? Eur J Clin Nutr. 2004;58(2):258-63. doi:10.1038/sj.ejen.1601775.

11. Cleland V, Worsley A, Crawford D. What are the grade 5 and 6 children buying from school canteens and what do parents and teachers think? Nutr Diet. 2004;61(3):145-50.

12. Ratnayake N, Ekanayake L. Soft drink consumption in Sri Lankan adolescents. Public Health Nutr. 2012;15(8):1333-7. doi:10.1017/S1368980012001061. 